\title{
Angular and linear momentum of excited ferromagnets
}

\author{
Peng Yan, ${ }^{1}$ Akashdeep Kamra, ${ }^{1}$ Yunshan Cao, ${ }^{1}$ and Gerrit E. W. Bauer ${ }^{2,1}$ \\ ${ }^{1}$ Kavli Institute of NanoScience, Delft University of Technology, Lorentzweg 1, 2628 CJ Delft, The Netherlands \\ ${ }^{2}$ Institute for Materials Research and WPI-AIMR, Tohoku University, Sendai 980-8577, Japan
}

(Received 12 July 2013; published 14 October 2013)

\begin{abstract}
The angular momentum vector of a Heisenberg ferromagnet with isotropic exchange interaction is conserved, while under uniaxial crystalline anisotropy the projection of the total spin along the easy axis is a constant of motion. Using Noether's theorem, we prove that these conservation laws persist in the presence of dipole-dipole interactions. However, spin and orbital angular momentum are no longer conserved separately. We also define the linear momentum of ferromagnetic textures. We illustrate the general principles with special reference to spin transfer torques and identify the emergence of a nonadiabatic effective field acting on domain walls in ferromagnetic insulators.
\end{abstract}

DOI: 10.1103/PhysRevB.88.144413

PACS number(s): 75.30.Ds, 75.60.Ch, 85.75.-d

Mathematics can be very effective in guiding research when physical intuition fails, even in applied sciences such as condensed matter physics. An important tool is Noether's theorem ${ }^{1}$ and its generalizations $s^{2,3}$ that help identify invariants or continuity equations starting from the fundamental symmetry properties of a given system. In the field of spintronics, for instance, Noether's theorem has been used to express the spin current, i.e., the flow of spin angular momentum, ${ }^{4}$ in spinorbit-coupled systems. ${ }^{5}$ In metallic ferromagnets spin currents are carried by an imbalance between up-spin and down-spin electron currents and therefore accompanied by long-distance mass motion and strong Joule heating. Spin currents can also be carried by spin waves (magnons), thereby dissipating much less energy in some magnetic insulators with high crystal quality. ${ }^{4}$ Magnon-mediated spin transport in various systems has received some attention in recent years. ${ }^{6-9}$ Schütz et al. ${ }^{10}$ demonstrated that magnons in a mesoscopic Heisenberg ring generate a persistent spin current under an inhomogeneous magnetic field. In magnetization textures particle-based ${ }^{11-13}$ as well as magnonic spin currents ${ }^{14-17}$ cause spin transfer torques that induce magnetization dynamics such as a domain wall (DW) motion. Direct imaging of a domain wall motion induced by thermally induced magnonic spin currents has been reported by Jiang et al. ${ }^{18}$ The spin transfer torque in magnetic insulators is usually ascribed to conservation of spin angular momentum, implicitly assuming that the exchange interaction is isotropic. However, whereas a negative domain wall velocity, i.e., opposite to the spin-wave propagation direction, is the signature of a magnonic spin transfer torque, ${ }^{15-17}$ positive domain wall velocities were found in micromagnetic simulations. ${ }^{19-23}$ A conclusive explanation of the latter observation is still lacking. Even the spin current and the corresponding continuity equation in Heisenberg magnets has not yet been properly formulated, ${ }^{24,25}$ despite the proven angular momentum conservation in isolated magnetic systems. ${ }^{25}$

Tatara and Kohno ${ }^{12}$ predicted domain wall motion by the force or (linear) momentum transfer experienced by narrow domain walls at which electron spins are reflected. But Volovik ${ }^{26}$ noted that the linear momentum of magnetization dynamics is not invariant under spin rotations ${ }^{27}$ and explained this paradox by considering a dynamic equation for the spin degrees of freedom supplemented by a kinetic equation for the underlying incoherent fermionic excitations. ${ }^{26,28}$ However, this approach fails for ferromagnetic insulators, illustrating the need for a full understanding of linear and angular momentum transport in ferromagnets.

In this paper, we formulate the angular and linear momentum of excited ferromagnets based on Noether's theorem. ${ }^{1}$ Starting with the Landau-Lifshitz equation for magnetization dynamics and Maxwell's equations for dipolar fields, we provide a systematic formulation of the conservation laws for the rotational and translational motion of spin excitations, e.g., magnons or domain walls, based on general symmetry principles. We show that in the presence of magnetic dipole-dipole interactions the spin current is not conserved, only the total angular momentum composed of spin and orbital component is. Noether's theorem also guides us to a proper formulation of linear momentum in ferromagnets that identifies the nondissipative linear momentum transfer ("effective field") mechanism in magnetic textures.

The semiclassical dynamics of a ferromagnet is described by the Landau-Lifshitz equation

$$
\frac{\partial \mathbf{M}}{\partial t}=-\mathbf{M} \times \mathbf{H}_{\mathrm{eff}},
$$

where $\mathbf{M}=\left(M_{x}, M_{y}, M_{z}\right)$ is the magnetization vector with modulus $M_{0}=|\mathbf{M}|$ and $\mathbf{H}_{\text {eff }}=-\delta E / \delta \mathbf{M}$ is the effective field expressed as the variational derivative of the energy $E=\int \mathcal{H} d V$. The energy density

$$
\mathcal{H}=\frac{J}{2}(\nabla \mathbf{M})^{2}+f\left(M_{z}\right)-\mathbf{M} \cdot \mathbf{h}-\frac{\mathbf{h}^{2}}{8 \pi}
$$

consists of the exchange interaction and the magnetic-dipole interaction expressed by the field $\mathbf{h}$, and we chose here an easy uniaxial anisotropy $f$ along the $z$ axis. h obeys Maxwell's equations but for slow modulations considered here the magnetostatic approximation suffices: ${ }^{29}$

$$
\boldsymbol{\nabla} \times \mathbf{h}=0 ; \quad \nabla \cdot(\mathbf{h}+4 \pi \mathbf{M})=0 .
$$

We can write the Lagrangian density of the system as

$$
\mathcal{L}=M_{z} \dot{\phi}+\mathcal{H},
$$

where $\phi=\arctan \left(M_{y} / M_{x}\right)$ is the azimuthal angle of $\mathbf{M}$. With $\mathbf{h}=\nabla \psi$, the first of Eqs. (3) is satisfied identically, while Eq. (1) and the second equation of (3) are the Euler-Lagrange 
equations

$$
\frac{\partial}{\partial x_{i}} \frac{\partial \mathcal{L}}{\partial\left(\partial q / \partial x_{i}\right)}=\frac{\partial \mathcal{L}}{\partial q},
$$

where $q=M_{z}, \phi, \psi, \quad i=\{1, \ldots, 4\}, \quad x_{1,2,3}=x, y, z, \quad$ and $x_{4}=t$.

We can now construct field invariants, i.e., a combination of the fields and their derivatives as functions of time and space that is conserved in time. ${ }^{30}$ According to Noether's theorem any continuous transformation of coordinates under which the variation of the action vanishes generates a definite invariant. It should be noted that our results do not depend on the particular version of Noether's theorem ${ }^{2,3,30}$ but lead to exactly the same conserving densities and their currents. We employ the global symmetries to obtain conservation laws for a closed system containing a magnetization texture and the associated dipolar field. As a direct consequence, mechanisms for orbital and nonadiabatic spin angular momentum transfer torques are predicted.

Translational symmetry. Spatial translational invariance leads to the conservation of linear momentum while a time-translation symmetry gives rise to energy conservation. Application of Noether's theorem leads to the continuity equation $\partial T_{i k} / \partial x_{k}=0$ for the energy-momentum tensor

$$
T_{i k}=\left(\frac{\partial q}{\partial x_{i}} \frac{\partial}{\partial\left(\partial q / \partial x_{k}\right)}-\delta_{i k}\right) \mathcal{L},
$$

which can be derived from the invariance of the action under the spatiotemporal translation transformations $\delta x_{i}=\delta_{i j} \delta \epsilon_{j}$ and $\delta M_{z}=\delta \phi=\delta \psi=0$, where $\delta_{i j}$ is the Kronecker function and $\delta \epsilon_{j}$ are infinitesimal translations. $-T_{44}$ is the energy density and $T_{i 4}$ the linear momentum density,

$$
p_{i}=T_{i 4}=M_{z} \frac{\partial \phi}{\partial x_{i}} .
$$

Hence, the total energy $E=-\int T_{44} d V$ and the linear momentum

$$
P_{i}=\int T_{i 4} d V
$$

are conserved.

This conservation law is complicated by the nondifferentiability of the azimuthal angle $\phi$ at the north $(\theta=0)$ and south poles $(\theta=\pi){ }^{31}$ By parametrizing the spin variables in terms of $\left(M_{x}, M_{y}, M_{z}\right)$, the momentum density $(\cos \theta-1) \frac{\partial \phi}{\partial x_{i}}$ can be written as $\mathbf{A} \cdot \frac{\partial \mathbf{M}}{\partial x_{i}}$, where $\mathbf{A}=\left(M_{y} \mathbf{e}_{x}-M_{x} \mathbf{e}_{y}\right) / M_{0}\left(M_{0}+M_{z}\right)$ diverges on the line specified by the equations $M_{x}=M_{y}=0$ and $M_{z}=-M_{0}$ (south pole). The singularity can be removed by employing an arbitrariness in the Lagrangian (4) that can be written as $\mathcal{L}=\left(C+M_{z}\right) \dot{\phi}+\mathcal{H}$, where the choice of the constant $C$ does not affect the dynamics, although a given $C$ cannot remove the singularities at two poles simultaneously.

The dynamic part of the Lagrangian (4) in terms of $\mathbf{M}$ and $\partial \mathbf{M} / \partial t$ coincides with that of a charged particle in a nonsingular magnetic field $\mathbf{B}=\boldsymbol{\nabla} \times \mathbf{A}=-\mathbf{M} / M_{0}^{3}$ for $M_{0} \neq 0$, in terms of a vector potential A. Varying $C$ is therefore equivalent to a gauge transformation. The linear momentum $p_{i}=\left(C+M_{z}\right) \partial \phi / \partial x_{i}$ is not invariant under these gauge transformations, but the difference between momenta of distinguishable states is. We illustrate this notion by the

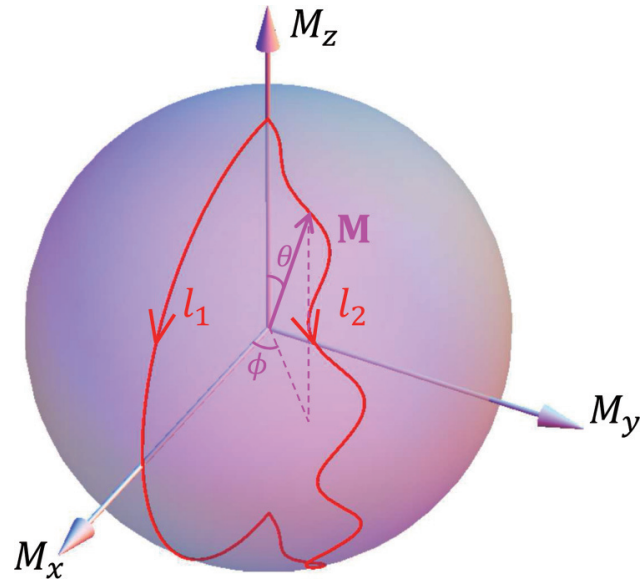

FIG. 1. (Color online) The Bloch sphere $|\mathbf{M}|=M_{0}$ with trajectories through domain walls with various configurations. Trajectory $l_{1}$ indicates a planar domain wall in the $y-z$ plane, while $l_{2}$ describes a general domain wall structure with local twists. The area of the contour $l_{2} \bar{l}_{1}$ on the sphere specifies the difference in the domain wall momentum described by trajectories $l_{2}$ and $l_{1}$.

momentum of a $180^{\circ}$ magnetic domain wall that is determined by path integrals of the form $M_{0} \int \mathbf{A} \cdot d \mathbf{M}$ along a trajectory connecting $M_{z}=-M_{0}$ and $M_{z}=+M_{0}$ (see $l_{1}$ or $l_{2}$ in Fig. 1$)$. The difference between the momenta is governed by the integral $M_{0} \int \mathbf{A} \cdot d \mathbf{M}$ along a closed contour, i.e., path $l_{2} \bar{l}_{1}$. According to Stokes theorem, the integral in question can be represented as the flux of the vector $\mathbf{B}=\boldsymbol{\nabla} \times \mathbf{A}$ through the surface bounded by this contour,

$$
\Delta P_{2-1}=M_{0} \iint_{S_{2-1}} \mathbf{B} \cdot d \mathbf{S},
$$

which is now gauge invariant. The spin electromotive force for electrons in moving magnetization textures is expressed by a similar contour integral. ${ }^{32}$ Here we express the domain wall (DW) momentum as

$$
P_{\mathrm{DW}}=M_{0} \iint \sin \theta d \theta d \phi,
$$

where the momentum of a fully in-plane DW $(\phi=0)$ defines the zero. When the DW plane is not twisted, the above equation leads to $P_{\mathrm{DW}}=2 \phi M_{0}$. The linear momentum carried by a rigid DW depends only on the tilt angle $\phi$ of its plane. The conclusion that even a completely static domain wall has a finite linear momentum is counterintuitive but can be rationalized in terms of the persistent angular momentum current generated by this topological defect.

Let us now consider the reflection of a spin wave with wave vector $\mathbf{k}$ by a planar domain wall as illustrated in Fig. 2(a). According to Eq. (8), the total linear momentum should be conserved during the scattering process,

$$
0=\frac{d \mathbf{P}}{d t}=\frac{d}{d t}\left(\mathbf{P}_{\mathrm{SW}}+\mathbf{P}_{\mathrm{DW}}\right),
$$

where $\mathbf{P}_{\mathrm{SW}}$ and $\mathbf{P}_{\mathrm{DW}}$ are the momenta of the spin wave and domain wall, respectively. Therefore, we have

$$
\mathbf{F}_{\mathrm{DW}}=2 M_{0} \dot{\phi} \mathbf{e}_{y},
$$




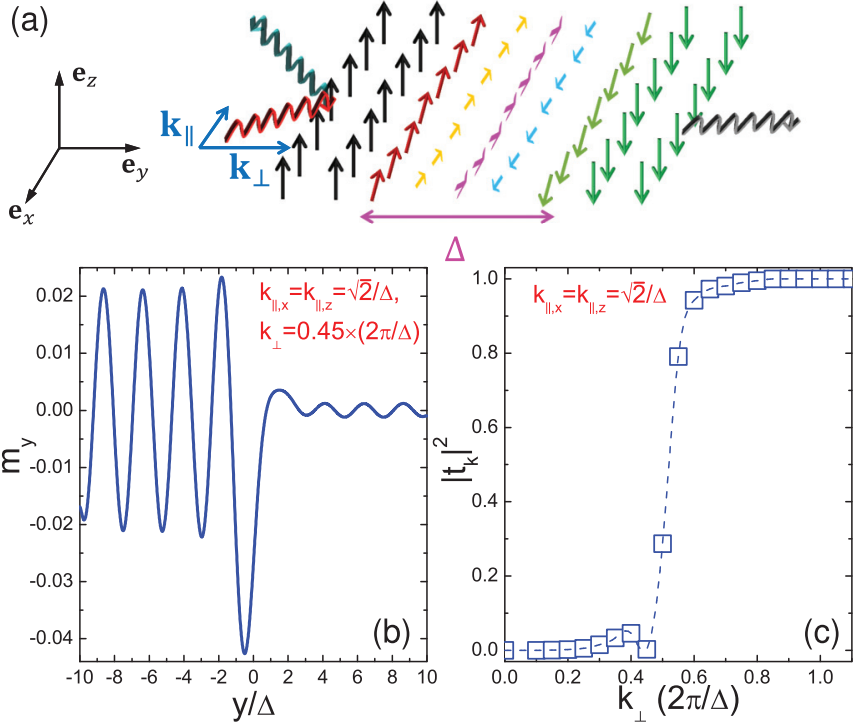

FIG. 2. (Color online) (a) Illustration of spin waves (wavy lines with arrows, red for incoming, aqua for reflection, and gray for transmission) scattered by a Bloch wall. $\mathbf{k}_{\|}$and $\mathbf{k}_{\perp}$ stand for the wave vectors parallel and perpendicular to the domain wall plane, respectively. $\Delta$ is the domain wall width. (b) Spin-wave reflection by a DW in three dimensions (3D) with $\mathbf{k}_{\|}=(\sqrt{2} / \Delta)\left(\mathbf{e}_{x}+\mathbf{e}_{z}\right)$ and $\mathbf{k}_{\perp}=0.45 \times(2 \pi / \Delta) \mathbf{e}_{y}$ obtained by solving the linearized LandauLifshitz equation coupled with Maxwell's equation (Ref. 33). The anisotropy energy is $f\left(M_{z}\right)=-(1 / 2) K M_{z}^{2}$ with $K / 4 \pi=0.01$. (c) $k_{\perp}$ dependence of SW transmission $\left|t_{k}\right|^{2}$ for a fixed $\mathbf{k}_{\|}$.

where $\mathbf{F}_{\mathrm{DW}}=d \mathbf{P}_{\mathrm{DW}} / d t$ is the force transferred by the spin wave to the domain wall by reflection. We find that spin-wave reflection is possible only under simultaneous rotation of the domain wall. Vice versa, linear momentum transfer does not lead to linear motion of the domain wall, but a rotation of the domain wall plane. Linear momentum transfer is thereby shown to be equivalent to an "effective" Zeeman magnetic field. Note that when the axial symmetry is broken, spin-wave reflection without coherent rotation becomes possible.

When including energy dissipation in the form of Gilbert damping $\alpha \mathbf{M} \times \dot{\mathbf{M}} / M_{0}$ by a collective coordinate approach and Walker ansatz, ${ }^{34}$ we find that rotation of the DW plane induced by spin-wave reflection is associated with linear propagation along the same direction as the spin wave. Its velocity $\mathbf{v}_{\text {DW }}=\alpha \Delta \dot{\phi} \mathbf{e}_{y}$, where $\Delta$ is the DW width. We thereby solve the puzzles mentioned in the Introduction. ${ }^{19-23}$ Spin-wave reflection at a domain wall is per definition a "non-adiabatic" process. The resultant torque due to this linear momentum transfer is nondissipative, and thereby different from the dissipative one proposed by Kovalev and Tserkovnyak. ${ }^{17}$ Unlike the dissipative correction for the adiabatic limit of wide domain walls, ${ }^{17}$ the nondissipative one predicted here is significant for domain walls subject to spin waves with non-normal incidence $\left(k_{\|} \neq 0\right)$ and $k_{\perp} \Delta \lesssim 0.1$. The latter statement requires some qualification. Spin-wave reflection predicted in a $1 \mathrm{D}$ spin chain $^{35}$ (equivalent to a normal-incidence spin wave in higher dimensions) requires atomic pinning by the discrete lattice, an effect that is beyond the continuum model used here. However, for $k_{\|} \neq 0$ and sharp domain walls the dipolar interaction leads to strong reflection also in the continuum model as is illustrated by the computed spin-wave amplitudes in Fig. 2(b), while the $k_{\perp}$ dependence of spin wave transmission probabilities is presented in Fig. 2(c). ${ }^{33}$ The dissipative correction favours a negative DW velocity (opposite to $\mathbf{k}_{\perp}$ ), ${ }^{17}$ while the nondissipative nonadiabatic torque leads to a positive one. The two mechanisms can be distinguished in a series of experiments or simulations on magnetic wires in which either the domain wall widths or the wavelengths of injected magnons are tunable.

Rotational symmetry. According to Noether's theorem the axial symmetry under spatial rotation around the easy $z$ axis, also in the presence of magnetostatic dipole-dipole interaction, implies conservation of angular momentum in this direction. A rotation around the $z$ axis is generated by $\delta x=y \delta \epsilon, \delta y=$ $-x \delta \epsilon, \delta z=\delta M_{z}=\delta \psi=0$, and $\delta \phi=-\delta \epsilon$, where $\delta \epsilon$ is the infinitesimal rotation parameter around the $z$ axis. Defining the $z$ component of the angular momentum current density

$$
\begin{aligned}
j_{z \mu}= & -J\left(M_{0}^{2}-M_{z}^{2}\right) \frac{\partial \phi}{\partial x_{\mu}}+J\left(M_{0}^{2}-M_{z}^{2}\right) \frac{\partial \phi}{\partial x_{\mu}}(\mathbf{r} \times \nabla \phi)_{z} \\
& +J \frac{M_{0}^{2}}{M_{0}^{2}-M_{z}^{2}} \frac{\partial M_{z}}{\partial x_{\mu}}\left(\mathbf{r} \times \nabla M_{z}\right)_{z}-M_{\mu}(\mathbf{r} \times \nabla \psi)_{z} \\
& -\frac{1}{4 \pi} \frac{\partial \psi}{\partial x_{\mu}}(\mathbf{r} \times \nabla \psi)_{z}-\varepsilon_{\mu z \nu} x_{\nu} \mathcal{L},
\end{aligned}
$$

where $\varepsilon_{\mu z v}$ is the Levi-Cività symbol and $\mu=\{1,2,3\}$, as well as

$$
j_{z 4}=-M_{z}+M_{z}(\mathbf{r} \times \nabla \phi)_{z},
$$

Noether's theorem leads us to the conservation law for the angular momentum along the $z$ axis,

$$
\frac{\partial j_{z 4}}{\partial t}+\frac{\partial j_{z \mu}}{\partial x_{\mu}}=0 .
$$

The first term in Eq. (14) is the spin angular momentum density, ${ }^{15}$ while the second one can be identified as an orbital angular momentum density since it can be written as $(\mathbf{r} \times \mathbf{p})_{z}$ where $\mathbf{p}=M_{z} \boldsymbol{\nabla} \phi$ is the linear momentum density obtained before [Eq. (7)]. Noether's theorem states that $\int\left(\partial j_{z \mu} / \partial x_{\mu}\right) d V=0$. Specifically, the $z$ component of the total angular momentum,

$$
J_{z}=\int j_{z 4} d V
$$

is conserved, which is consistent with the result in a previous paper. ${ }^{25}$ However, the angular momentum current [Eq. (13)] and its continuity equation [Eq. (15)] can be obtained only through our very general and systematic approach.

Conservation of spin angular momentum has been discussed for purely exchange-coupled ferromagnets. ${ }^{14,15}$ In the presence of magnetic dipolar interactions, the energymomentum or stress tensor $T_{i k}$ becomes nonsymmetric and the orbital angular momentum

$$
L_{i}=\int \varepsilon_{i j k} x_{j} T_{k 4} d V, \quad i=\{1,2,3\},
$$

does not vanish. The $z$ projection of the integrand, i.e., the orbital angular momentum density, agrees with the second term of Eq. (14). Since $d L_{i} / d t=-\int \varepsilon_{i j k} M_{j}\left(\partial \psi / \partial x_{k}\right) d V \neq$ 
0 , a nonzero $L_{i}$ is then not conserved. We note the analogy with the coupling of spins by the spin-orbit interaction of electrons in the weakly relativistic limit, a role that is played here by the dipole-dipole interaction. We note that the angular momentum density of the electromagnetic field $\vec{j}=\mathbf{r} \times(\mathbf{e} \times \mathbf{h})($ Ref. 36) is negligibly small in the regime where the magnetostatic approximation [as in Eq. (3)] holds, in which the electric field e plays no role whatsoever. ${ }^{29}$

We now illustrate our results [Eq. (14)] for a uniform ferromagnetic nanocylinder $\left(\mathbf{M}=M_{0} \mathbf{e}_{z}\right)$ with uniaxial anisotropy $f\left(M_{z}\right)=-\frac{1}{2} K M_{z}^{2}$ and spin-wave excitation $\mathbf{m}=\left(m_{x}, m_{y}, 0\right)$ $\left(\mathbf{M}=M_{0} \mathbf{e}_{z}+\mathbf{m}\right)$. The Hamiltonian to leading order in $\mathbf{m}$ is diagonalized by the Bogoliubov transformation

$$
\begin{aligned}
m_{+} & =m_{x}+i m_{y} \\
& =\sqrt{\frac{2 \hbar M_{0}}{V}} \sum_{\mathbf{k}}\left\{u_{\mathbf{k}} a_{\mathbf{k}} e^{i\left(\mathbf{k} \cdot \mathbf{r}-\omega_{\mathbf{k}} t\right)}+v_{\mathbf{k}}^{*} a_{\mathbf{k}}^{\dagger} e^{-i\left(\mathbf{k} \cdot \mathbf{r}-\omega_{\mathbf{k}} t\right)}\right\},
\end{aligned}
$$

where $a_{\mathbf{k}}^{\dagger}$ and $a_{\mathbf{k}}$ are Bose creation and annihilation operators while the coefficients $u_{\mathbf{k}}$ and $v_{\mathbf{k}}$ and the frequency $\omega_{\mathbf{k}}$ are related by the equations $A_{\mathbf{k}} u_{\mathbf{k}}+$ $B_{\mathbf{k}}^{*} v_{\mathbf{k}}=\omega_{\mathbf{k}} u_{\mathbf{k}}, B_{\mathbf{k}} u_{\mathbf{k}}+A_{\mathbf{k}} v_{\mathbf{k}}=-\omega_{\mathbf{k}} v_{\mathbf{k}}$, and $\left|u_{\mathbf{k}}\right|^{2}-\left|v_{\mathbf{k}}\right|^{2}=$ 1 , with $A_{\mathbf{k}}=J M_{0} k^{2}+K M_{0}+2 \pi M_{0}\left(k_{x}^{2}+k_{y}^{2}\right) / k^{2}, B_{\mathbf{k}}=$ $2 \pi M_{0}\left(k_{x}+i k_{y}\right)^{2} / k^{2}$, and $\mathcal{H}=\mathcal{H}_{0}+\sum_{\mathbf{k}} \hbar \omega_{\mathbf{k}} a_{\mathbf{k}}^{\dagger} a_{\mathbf{k}}$ with $\omega_{\mathbf{k}}=$ $\sqrt{A_{\mathbf{k}}^{2}-\left|B_{\mathbf{k}}\right|^{2}}$. The total angular momentum Eq. (16) reads

$$
J_{z}-J_{z 0}=-\hbar \sum_{\mathbf{k}} a_{\mathbf{k}}^{\dagger} a_{\mathbf{k}}-i \hbar \sum_{\mathbf{k}} a_{\mathbf{k}}^{\dagger}\left(\mathbf{k} \times \nabla_{\mathbf{k}}\right) a_{\mathbf{k}},
$$

where $\nabla_{\mathbf{k}}$ is the gradient in $\mathbf{k}$ space. The first and second terms on the right-hand side are the spin and orbital angular momenta of the magnon excitations, respectively. By transforming from Cartesian to cylindrical coordinates $\rho, k_{z}$, and $n$, and by $a_{\mathbf{k}}=$ $\sum_{n} a_{\rho, k_{z}, n} e^{i n \phi}$, where $\rho=\sqrt{k_{x}^{2}+k_{y}^{2}}$ and $n$ is the azimuthal quantum number [the eigenvalue of the operator $i\left(\mathbf{k} \times \nabla_{\mathbf{k}}\right)_{z}=$ $\left.i \frac{\partial}{\partial \phi}\right], J_{z}-J_{z 0}=\hbar \sum_{n}(n-1) a_{\rho, k_{z}, n}^{\dagger} a_{\rho, k_{z}, n}$. The total angular momentum therefore depends on the magnetization profile in the transverse plane. Different $n$ correspond to different wave-front shapes of the helical (vortex) spin wave modes. The dipole-exchange spin waves in cylindrical ferromagnetic nanowires display a rich wave pattern in the cross section of the nanowire. ${ }^{37}$ Vortex modes with high orbital angular momentum have been achieved in photonic ${ }^{38}$ and electronic ${ }^{39}$ wave guides using spiral phase plates, computer-generated holograms, etc. It should be very interesting to generate helical spin-wave modes and realize the conversion of angular momentum from orbit to spin experimentally. Spin waves with high orbital angular momenta would be efficient drivers of domain wall motion in the axially symmetric nanocylinders that have been successfully fabricated and imaged recently. ${ }^{40}$ The natural magnon mode in a cylindrical domain carries an orbital angular momentum $n \hbar$. Spin waves propagating through a domain wall accumulate phase shifts ${ }^{15}$ corresponding to an orbital angular momentum $n^{\prime} \hbar$ of transmitted waves $\left(n \neq n^{\prime}\right.$ for complex wall structures in the presence of dipole-dipole interactions). The transfer of orbital angular momentum is enhanced for large $\left|n-n^{\prime}\right|$, leading to efficient domain wall motion.

To conclude, we formulate the conservation laws of linear and angular momenta in ferromagnetic textures in the presence of magnetic dipole-dipole interactions based on Noether's theorem. We derive a well-defined linear momentum for insulating ferromagnets without involving incoherent fermionic excitations, thereby resolving a paradox raised by Volovik. ${ }^{26}$ Mathematics helps to correct misguided physical intuition that naively associates linear momentum to domain wall translational motion. Instead, we show that linear momentum transfer of spin waves reflected at magnetic domain walls induces an effective field and steady rotation of the domain wall plane rather than translation. Only in the presence of dissipation does this lead to domain wall propagation. Besides the usual spin angular momentum, we identity an orbital angular momentum of spin waves that is linked to the shapes of their wave fronts. We expect to stimulate experiments on the preparation and manipulation of spin waves, thereby opening an additional research direction in the field of magnonics.

This work is supported by the FOM foundation, DFG Priority Program 1538 SpinCat, EG-STREP MACALO, MarieCurie ITN Spinicur, and Grants-in-Aid for Scientific Research A (Kakenhi) No. 25247056.
${ }^{1}$ E. Noether, Nachr. Ges. Wiss. Göttingen 235 (1918).

${ }^{2}$ P. J. Olver, Applications of Lie Groups to Differential Equations (Springer, New York, 2000).

${ }^{3}$ N. H. Ibragimov, Transformation Groups Applied to Mathematical Physics (D. Reidel, Dordrecht, 2001).

${ }^{4}$ Spin Current, edited by S. Maekawa, S. O. Valenzuela, E. Saitoh, and T. Kimura (Oxford University Press, Oxford, 2012).

${ }^{5}$ Y. Wang, K. Xia, Z. B. Su, and Z. S. Ma, Phys. Rev. Lett. 96, 066601 (2006).

${ }^{6}$ M. Takigawa, N. Motoyama, H. Eisaki, and S. Uchida, Phys. Rev. Lett. 76, 4612 (1996).

${ }^{7}$ X. Zotos, Phys. Rev. Lett. 82, 1764 (1999).

${ }^{8}$ J. V. Alvarez and C. Gros, Phys. Rev. Lett. 88, 077203 (2002).

${ }^{9}$ F. Meier and D. Loss, Phys. Rev. Lett. 90, 167204 (2003).
${ }^{10}$ F. Schütz, M. Kollar, and P. Kopietz, Phys. Rev. Lett. 91, 017205 (2003); Phys. Rev. B 69, 035313 (2004).

${ }^{11}$ S. S. P. Parkin, M. Hayashi, and L. Thomas, Science 320, 190 (2008).

${ }^{12}$ G. Tatara and H. Kohno, Phys. Rev. Lett. 92, 086601 (2004).

${ }^{13}$ S. Zhang and Z. Li, Phys. Rev. Lett. 93, 127204 (2004).

${ }^{14}$ A. V. Mikhailov and A. I. Yaremchuk, Pis'ma Zh. Eksp. Teor. Fiz. 39, 296 (1984) [JETP Lett. 39, 354 (1984)].

${ }^{15}$ P. Yan, X. S. Wang, and X. R. Wang, Phys. Rev. Lett. 107, 177207 (2011).

${ }^{16}$ D. Hinzke and U. Nowak, Phys. Rev. Lett. 107, 027205 (2011).

${ }^{17}$ A. A. Kovalev and Y. Tserkovnyak, Europhys. Lett. 97, 67002 (2012). 
${ }^{18}$ W. J. Jiang, P. Upadhyaya, Y. B. Fan, J. Zhao, M. S. Wang, L. T. Chang, M. R. Lang, K. L. Wong, M. Lewis, Y. T. Lin, J. S. Tang, S. Cherepov, X. Z. Zhou, Y. Tserkovnyak, R. N. Schwartz, and K. L. Wang, Phys. Rev. Lett. 110, 177202 (2013).

${ }^{19}$ D. S. Han, S. K. Kim, J. Y. Lee, S. J. Hermsdoerfer, H. Schultheiss, B. Leven, and B. Hillebrands, Appl. Phys. Lett. 94, 112502 (2009).

${ }^{20}$ M. Jamali, H. Yang, and K. J. Lee, Appl. Phys. Lett. 96, 242501 (2010).

${ }^{21}$ S. M. Seo, H. W. Lee, H. Kohno, and K. J. Lee, Appl. Phys. Lett. 98, 012514 (2011).

${ }^{22}$ J. S. Kim, M. Stärk, M. Kläui, J. Yoon, C. Y. You, L. Lopez-Diaz, and E. Martinez, Phys. Rev. B 85, 174428 (2012).

${ }^{23}$ X. G. Wang, G. H. Guo, Y. Z. Nie, G. F. Zhang, and Z. X. Li, Phys. Rev. B 86, 054445 (2012).

${ }^{24}$ F. Schütz, P. Kopietz, and M. Kollar, Eur. Phys. J. B 41, 557 (2004).

${ }^{25}$ Y. Gaididei, V. P. Kravchuk, D. D. Sheka, and F. G. Mertens, Phys. Rev. B 81, 094431 (2010).

${ }^{26}$ G. E. Volovik, J. Phys. C 20, L83 (1987).

${ }^{27}$ F. D. M. Haldane, Phys. Rev. Lett. 57, 1488 (1986).

${ }^{28}$ C. H. Wong and Y. Tserkovnyak, Phys. Rev. B 80, 184411 (2009).
${ }^{29}$ D. D. Stancil, Theory of Magnetostatic Waves (Springer, New York, 1993).

${ }^{30}$ N. N. Bogolyubov and D. V. Shirkov, Introduction to the Theory of Quantized Fields (Interscience, New York, 1959).

${ }^{31}$ N. Papanicolaou and T. N. Tomaras, Nucl. Phys. B 360, 425 (1991).

${ }^{32}$ S. E. Barnes and S. Maekawa, Phys. Rev. Lett. 98, 246601 (2007).

${ }^{33}$ Detailed calculations will be presented elsewhere.

${ }^{34}$ N. L. Schryer and L. R. Walker, J. Appl. Phys. 45, 5406 (1974).

${ }^{35}$ P. Yan and G. E. W. Bauer, Phys. Rev. Lett. 109, 087202 (2012); IEEE Trans. Magn. 49, 3109 (2013).

${ }^{36}$ L. Allen, S. M. Barnett, and M. J. Padgett, Optical Angular Momentum (Institute of Physics Publishing, Bristol, 2003).

${ }^{37}$ R. Arias and D. L. Mills, Phys. Rev. B 63, 134439 (2001); 66, 149903(E) (2002).

${ }^{38}$ X. L. Cai, J. W. Wang, M. J. Strain, B. J. Morris, J. B. Zhu, M. Sorel, J. L. O'Brien, M. G. Thompson, and S. Y. Yu, Science 338, 363 (2012).

${ }^{39}$ B. J. McMorran, A. Agrawal, I. M. Anderson, A. A. Herzing, H. J. Lezec, J. J. McClelland, and J. Unguris, Science 331, 192 (2011).

${ }^{40}$ N. Biziere, C. Gatel, R. L. Balier, M. C. Clochard, J. E. Wegrowe, and E. Snoeck, Nano Lett. 13, 2053 (2013). 